ARTICLE

Received 18 Jun 2014 | Accepted 24 Jul 2014 | Published 5 Sep $2014 \quad$ DOl: 10.1038/ncomms5802

\title{
Somatic mutations in DROSHA and DICER1 impair microRNA biogenesis through distinct mechanisms in Wilms tumours
}

Dinesh Rakheja ${ }^{1,2,3,4, \star}$, Kenneth S. Chen $2,5,6, \star$, Yangjian Liu ${ }^{5, *}$, Abhay A. Shukla ${ }^{2,5}$, Vanessa Schmid ${ }^{7}$, Tsung-Cheng Chang ${ }^{5}$, Shama Khokhar ${ }^{3}$, Jonathan E. Wickiser ${ }^{2,6}$, Nitin J. Karandikar', James S. Malter ${ }^{1}$, Joshua T. Mendell ${ }^{5,8}$ \& James F. Amatruda $2,4,5,6,8$

Wilms tumour is the most common childhood kidney cancer. Here we report the wholeexome sequencing of 44 Wilms tumours, identifying missense mutations in the microRNA (miRNA)-processing enzymes DROSHA and DICER1, and novel mutations in MYCN, SMARCA4 and ARID1A. Examination of tumour miRNA expression, in vitro processing assays and genomic editing in human cells demonstrates that DICER1 and DROSHA mutations influence miRNA processing through distinct mechanisms. DICER1 RNase IIIB mutations preferentially impair processing of miRNAs deriving from the $5^{\prime}$-arm of pre-miRNA hairpins, while DROSHA RNase IIIB mutations globally inhibit miRNA biogenesis through a dominant-negative mechanism. Both DROSHA and DICER1 mutations impair expression of tumour-suppressing miRNAs, including the let-7 family, important regulators of MYCN, LIN28 and other Wilms tumour oncogenes. These results provide new insights into the mechanisms through which mutations in miRNA biogenesis components reprogramme miRNA expression in human cancer and suggest that these defects define a distinct subclass of Wilms tumours.

\footnotetext{
${ }^{1}$ Department of Pathology, University of Texas Southwestern Medical Center, Dallas, Texas 75390, USA. ${ }^{2}$ Department of Pediatrics, University of Texas Southwestern Medical Center, Dallas, Texas 75390, USA. ${ }^{3}$ Division of Pathology and Laboratory Medicine, Children's Medical Center, Dallas, Texas 75235, USA. ${ }^{4}$ Harold C. Simmons Comprehensive Cancer Center, University of Texas Southwestern Medical Center, Dallas, Texas 75390, USA. ${ }^{5}$ Department of Molecular Biology, University of Texas Southwestern Medical Center, Dallas, Texas 75390, USA. ${ }^{6}$ Gill Center for Cancer and Blood Disorders, Children's Medical Center, Dallas, Texas 75235, USA. 7 Eugene McDermott Center for Human Growth and Development, University of Texas Southwestern Medical Center, Dallas, Texas 75390, USA. ${ }^{8}$ Department of Internal Medicine, University of Texas Southwestern Medical Center, Dallas, Texas 75390, USA.

* These authors contributed equally to this work. Correspondence and requests for materials should be addressed to J.T.M. (email: joshua.mendell@utsouthwestern.edu) or to J.F.A. (email: james.amatruda@utsouthwestern.edu).
} 
W ilms tumour is the most common childhood genitourinary tract cancer and the third most common paediatric solid tumour. It comprises $95 \%$ of all renal cancers and $6 \%$ of all cancers diagnosed among children $<15$ years of age ${ }^{1,2}$. Depending on tumour histology and disease stage, treatment consists of nephrectomy and combination chemotherapy, with or without radiotherapy. Although the overall survival of Wilms tumour patients has increased to $85 \%$ at 5 years after diagnosis ${ }^{3}$, children with diffusely anaplastic or unfavourable histology Wilms tumour continue to have poor outcomes, with 4 -year survival rates of $56 \%$ for Stage III and $17 \%$ for Stage $\mathrm{IV}^{4-6}$. Moreover, the success of modern therapy comes at a price in that up to $70 \%$ of Wilms tumour survivors develop chronic health problems as young adults, including but not limited to renal failure, congestive heart failure, interstitial pulmonary fibrosis, kyphoscoliosis, infertility, intestinal obstruction and second malignancies ${ }^{7-9}$.

Few targetable molecular lesions have been defined in Wilms tumours. Recurrent genetic changes in sporadic Wilms tumours have been described, including mutations in WTX, WT1, CTNNB1 and TP53. However, these mutations account for only about $1 / 3$ of Wilms tumours ${ }^{10}$. Children with DICER1 syndrome, who carry one germline-null allele of DICER1, are susceptible to tumours, including pleuropulmonary blastoma and Wilms tumour, suggesting that defects in microRNA (miRNA) processing may increase susceptibility to this disease $\mathrm{e}^{11-13}$. This idea has further been reinforced by the recent description of somatic heterozygous missense mutations in DICER1 (ref. 14) and DROSHA ${ }^{15}$ in Wilms tumours. However, the mechanisms through which heterozygous DICER1 and DROSHA mutations affect miRNA biogenesis in Wilms tumour are unknown and it is unclear whether mutations in these proteins have similar or distinct effects on miRNA expression in these tumours. Furthermore, it is unknown whether other genes are recurrently mutated in Wilms tumour beyond those already described.

Here we describe the results of whole-exome sequencing of a cohort of 44 Wilms tumours. In this study, we identify both known and novel recurrent, somatic mutations. We carry out functional studies in vitro and in genetically modified cell lines to elucidate the mechanisms by which missense mutations in DICER1 and DROSHA affect miRNA expression in tumours. These studies identify a new subclass of Wilms tumours and suggest that impaired expression of let-7 tumour-suppressing miRNAs may be a common underlying mechanism of this subclass.

\section{Results}

Exome sequencing of Wilms tumours. To discover the full range of pathogenic Wilms tumour mutations, we performed exome capture and massively parallel sequencing on a discovery set of 15 pairs of Wilms tumours and matched adjacent normal kidney cortices, and subsequently performed whole-exome sequencing on a validation set of 29 additional Wilms tumours. Table 1 lists the patient demographics and Supplementary Data 1 lists the total number of variants identified in the tumours. In the discovery set, we identified between 0 and 17 non-synonymous somatic single nucleotide variants per tumour (Fig. 1a and Supplementary Data 2). These numbers are consistent with the generally lower mutational load seen in paediatric tumours compared with tumours from adults ${ }^{16,17}$.

Mutations were identified in genes previously associated with Wilms tumour as well as in genes associated with other paediatric tumour types (Fig. 1b,d, and Supplementary Data 2 and 3). For example, seven Wilms tumours harboured mutations in either WT1, or CTNNB1, or both (Fig. 1b). Four of 44 (9\%) Wilms tumours had diffusely anaplastic histology, and three of these tumours harboured missense mutations of TP53, consistent with the known association of anaplastic Wilms tumours with TP53 mutation ${ }^{4}$. Somatic mutations in the chromatin-remodelling factors SMARCA4 and ARID1A, which are known to be mutated in other childhood cancers ${ }^{18-21}$, were each seen in two tumours (4.5\%). MYCN was mutated at codon 44 in two tumours (4.5\%), creating a P44L substitution at a highly conserved residue. The same mutation was recently reported in high-risk neuroblastoma ${ }^{22}$. MYCN is overexpressed in some Wilms tumour $^{23}$ through genomic amplification ${ }^{24}$ and through mutations in FBXW7, an E3 ubiquitin ligase that regulates MYCN stability ${ }^{25}$. All mutations listed in Figure $1 \mathrm{~b}$ and Supplementary Data 3 were confirmed by Sanger sequencing of tumour and germline DNA.

In addition to these known and novel mutations, we identified mutations in components of the miRNA biogenesis machinery in eight tumours in our cohort (18\%). Primary (pri)-miRNAs are processed in the nucleus by the RNase III enzyme DROSHA (in a complex with the double-stranded RNA binding protein DGCR8) to yield smaller pre-miRNAs, which are transported to the cytoplasm and processed by DICER1 (in a complex with TARBP2) to produce mature miRNAs ${ }^{26}$. Six Wilms tumours in our cohort showed heterozygous missense mutations in DROSHA (14\%); one of these tumours (CMCW39) also harboured a somatic D221G mutation in TARBP2. Another two tumours contained novel missense mutations in DICER1, and one of these tumours also harboured a germline frameshift deletion predicted to create a null allele (Fig. 1b-d). The frequency of mutations in miRNA pathway genes and specifically in DROSHA were overall similar to that reported by Torrezan et al. ${ }^{15}$ (33\% and $12 \%$, respectively). All DICER1 and DROSHA missense mutations were somatic with the exception of the DROSHA R967W mutation in CMCW41 and the DROSHA M120V mutation in CMCW53, which were also present in non-tumour tissue. One tumour (CMCW85) harbored a germline DGCR8 S720N variant. Pertinently, miRNA processing mutations were mutually exclusive from WT1 or CTNNB1 mutations, suggesting that impairment of miRNA biogenesis represents a novel mode of oncogenesis in a subset of Wilms tumours.

Functional analysis of miRNA processing mutations in vitro. DROSHA and DICER1 both possess two highly conserved tandem endonuclease domains, denoted RNase IIIA (RIIIA) and RNase IIIB (RIIIB) ${ }^{27}$. The RIIIA domains of DROSHA and DICER1 selectively process the $3^{\prime}$-arm of the pri-miRNA or the pre-miRNA hairpin, respectively, while the RIIIB domains selectively process the $5^{\prime}$-arm of the pri-miRNA or the premiRNA, respectively ${ }^{28,29}$ (Fig. 2a). Cleavage of double-stranded RNA is $\mathrm{Mg}^{2+}$-dependent and requires the presence of conserved metal-binding aspartate and glutamate residues in the RIIIA and RIIIB domains ${ }^{30,31}$. Recurrent somatic mutations in the metalbinding residues of the DICER1 RIIIB domain (E1705, D1709, D1810 and E1813) have been reported in sex cord-stromal cell tumours and germ cell tumours ${ }^{32}$. In vitro processing assays and cellular reconstitution experiments have demonstrated that mutations in metal-binding residues of the DICER1 RIIIB domain strongly impair maturation of $5 \mathrm{p}$ miRNAs (those derived from the $5^{\prime}$-arm of the pre-miRNA hairpin), yet still allow partial production of $3 \mathrm{p}$ miRNAs (derived from the $3^{\prime}$-arm of the pre-miRNA hairpin) ${ }^{28,32,33}$.

The DICER1 missense mutations that we identified in Wilms tumours occur at the highly conserved glycine 1809 residue adjacent to the metal-binding aspartate residue 1810 within the RIIIB domain (Fig. 1c). In the Giardia lamblia RNase III crystal 
Table 1 | Clinical characteristics of patients in this study.

\begin{tabular}{|c|c|c|c|c|c|c|}
\hline Sample ID & Gender & Age (years) & Morphology & Surgical stage & Overall stage & Site of metastasis, if any \\
\hline CMCW1 & $M$ & 5.1 & Diffuse anaplasia & 1 & 4 & Lung \\
\hline CMCW3 & $M$ & 2.0 & $\mathrm{FH}$ & 3 & 3 & Adrenal \\
\hline CMCW5 & $M$ & 9.1 & $\mathrm{FH}$ & 3 & 4 & Lung \\
\hline CMCW7 & $\mathrm{F}$ & 3.7 & $\mathrm{FH}$ & 2 & 2 & \\
\hline CMCW9 & $\mathrm{F}$ & 1.5 & $\mathrm{FH}$ & 2 & 2 & \\
\hline CMCW11 & $\mathrm{F}$ & 5.2 & Diffuse anaplasia & 3 & 4 & Lymph node, rib, lung \\
\hline CMCW13 & $\mathrm{F}$ & 2.9 & $\mathrm{FH}$ & 3 & 3 & \\
\hline CMCW15 & $\mathrm{F}$ & 5.1 & $\mathrm{FH}$ & 3 & 4 & Lung \\
\hline CMCW17 & $M$ & 4.2 & $\mathrm{FH}$ & 2 & 2 & \\
\hline CMCW19 & $M$ & 2.5 & $\mathrm{FH}$ & 3 & 3 & Lymph node \\
\hline CMCW21 & $\mathrm{F}$ & 0.9 & $\mathrm{FH}$ & 1 & 1 & \\
\hline CMCW23 & $M$ & 3.5 & Diffuse anaplasia & 2 & 2 & \\
\hline CMCW25 & $M$ & 2.8 & $\mathrm{FH}$ & 3 & 3 & \\
\hline CMCW27 & $\mathrm{F}$ & 3.4 & $\mathrm{FH}$ & 2 & 4 & Lung \\
\hline CMCW29 & $\mathrm{F}$ & 7.9 & $\mathrm{FH}$ & 1 & 1 & \\
\hline CMCW31 & M & 0.8 & $\mathrm{FH}$ & 1 & 1 & \\
\hline CMCW33 & $\mathrm{F}$ & 2.9 & $\mathrm{FH}$ & 2 & 2 & \\
\hline CMCW35 & $\mathrm{F}$ & 4.2 & $\mathrm{FH}$ & 1 & 1 & \\
\hline CMCW37 & $\mathrm{F}$ & 3.7 & $\mathrm{FH}$ & 2 & 2 & \\
\hline CMCW39 & $M$ & 1.7 & $\mathrm{FH}$ & 2 & 2 & \\
\hline CMCW41 & $M$ & 4.3 & $\mathrm{FH}$ & 2 & 5 & \\
\hline CMCW43 & $\mathrm{F}$ & 4.7 & Focal anaplasia & 3 & 3 & \\
\hline CMCW45 & $M$ & 7.2 & $\mathrm{FH}$ & 3 & 4 & Lung \\
\hline CMCW47 & $M$ & 3.4 & $\mathrm{FH}$ & 2 & 2 & \\
\hline CMCW49 & $M$ & 2.5 & $\mathrm{FH}$ & 2 & 2 & \\
\hline CMCW51 & $M$ & 0.8 & $\mathrm{FH}$ & 3 & 3 & \\
\hline CMCW53 & $M$ & 8.3 & $\mathrm{FH}$ & 3 & 4 & Lung \\
\hline CMCW57 & $M$ & 0.9 & $\mathrm{FH}$ & 3 & 3 & \\
\hline CMCW59 & $M$ & 3.5 & Diffuse anaplasia & 3 & 3 & \\
\hline CMCW61 & $\mathrm{F}$ & 1.2 & $\mathrm{FH}$ & 2 & 2 & \\
\hline CMCW63 & $M$ & 1.4 & $\mathrm{FH}$ & 3 & 3 & \\
\hline CMCW65 & $\mathrm{F}$ & 2.3 & $\mathrm{FH}$ & 1 & 1 & \\
\hline CMCW67 & $M$ & 1.6 & $\mathrm{FH}$ & 2 & 2 & \\
\hline CMCW69 & $\mathrm{F}$ & 0.7 & $\mathrm{FH}$ & 3 & 3 & \\
\hline CMCW71 & $M$ & 2.7 & $\mathrm{FH}$ & 3 & 3 & \\
\hline CMCW75 & $\mathrm{F}$ & 5.2 & $\mathrm{FH}$ & 3 & 4 & Lung \\
\hline CMCW77 & $M$ & 1.3 & $\mathrm{FH}$ & 1 & 1 & \\
\hline CMCW79 & $\mathrm{F}$ & 3.3 & $\mathrm{FH}$ & 2 & 4 & Lung \\
\hline CMCW81 & $\mathrm{F}$ & 2.6 & $\mathrm{FH}$ & 2 & 2 & \\
\hline CMCW83 & $\mathrm{F}$ & 8.0 & $\mathrm{FH}$ & 3 & 4 & Lung, liver \\
\hline CMCW85 & $M$ & 2.3 & $\mathrm{FH}$ & 2 & 4 & Lung \\
\hline CMCW87 & $F$ & 1.2 & $\mathrm{FH}$ & 1 & 1 & \\
\hline CMCW89 & $\mathrm{F}$ & 6.7 & $\mathrm{FH}$ & 3 & 4 & Heart/lung \\
\hline CMCW91 & $M$ & 3.5 & $\mathrm{FH}$ & 3 & 3 & \\
\hline
\end{tabular}

structure $^{34}$, the equivalent residue G719 is within $4 \AA$ of a neighbouring $\alpha$-helix, suggesting that the G1809V and G1809R mutations may distort the active-site geometry and impair enzyme function (Fig. 1e). To directly determine the effects of these mutations on DICER1 activity, in vitro pre-miRNA processing assays were performed (Fig. 2b and Supplementary Fig. 1a). The DICER1 mutation I1102fsdel is predicted to produce a truncation before the ribonuclease domains and, as expected, I1102fsdel has no detectable activity with any substrate (Fig. 2b). When processed by the DICER1 RIIIB mutants G1809R and G1809V, 5p miRNAs show accumulation of the expected aberrant processing product due to cleavage by the RIIIA domain but not the RIIIB domain. For some very efficiently processed $5 \mathrm{p}$ miRNAs, some mature miRNA is still produced by these mutants (let-7i and miR-122), whereas other 5p miRNAs are not measurably processed (miR-16-1 and miR-18a). For the tested $3 p$ miRNAs, the G1809R and G1809V mutants produced normal (miR-1-1) or reduced (miR-124-2) amounts of the mature miRNA. These data demonstrate that the G1809R and G1809V substitutions impair activity of the DICER1 RIIIB domain and, consistent with prior findings ${ }^{28,33}$, suggest that these mutations inhibit the production of $5 \mathrm{p}$ miRNAs more severely than $3 p$ miRNAs.

The somatic DROSHA mutations E1147K and D1151Y (analogous to the E1705 and D1709 positions of DICER1) alter a conserved RNase IIIB domain metal-binding residue, while the L728V mutation is not in an annotated functional domain. E1147K was also identified by Torrezan et al. ${ }^{15}$ as a mutational hotspot $^{15}$. In vitro pri-miRNA processing assays revealed that the DROSHA D1151Y and E1147K mutants are functional nulls for all pri-miRNA substrates tested, while the L728V mutations does not measurably impair enzyme activity (L728V is less active in this assay, but this variant reproducibly expresses at lower levels (Supplementary Fig. 1b), precluding definitive conclusions about its activity) (Fig. 2c). We also detected two DROSHA mutations that were present in the germline: R967W that is adjacent to the 
a

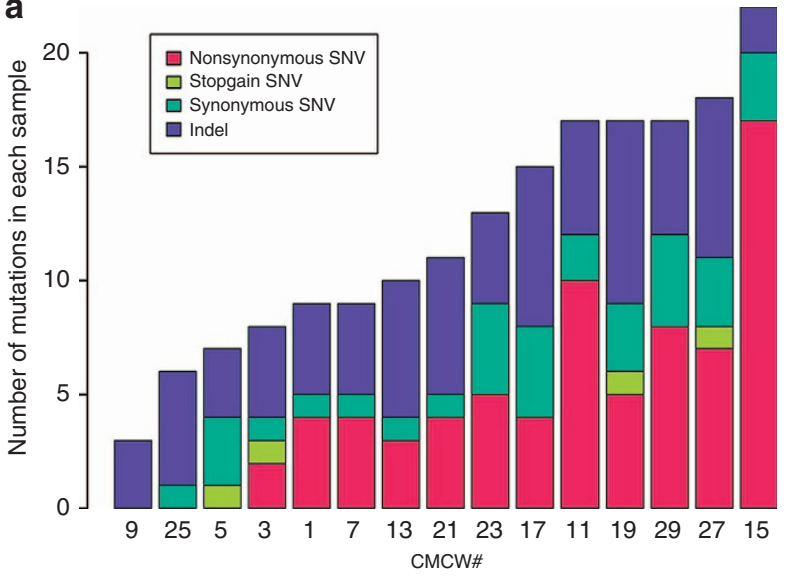

C

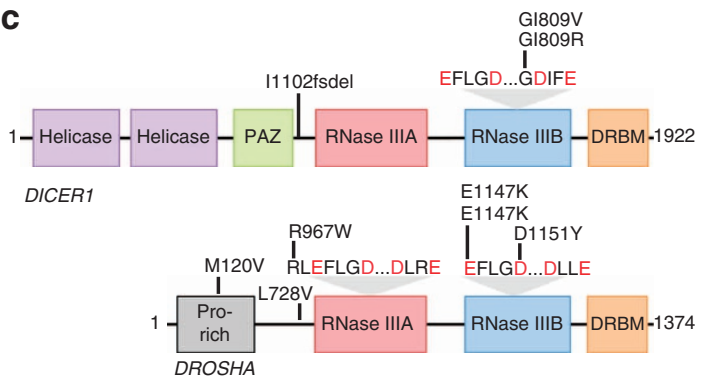

d

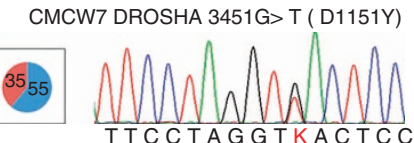

CMCW79 DROSHA 3439G>A (E1147K)

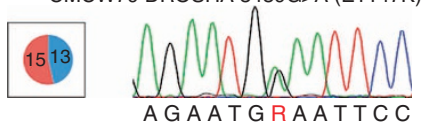

CMCW11 DICER1 5425G>A (G1809R)
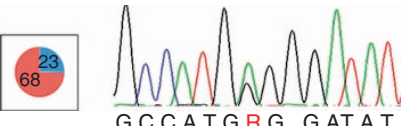

CMCW15 DROSHA 3439G>A ( E1147K)

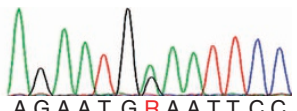

AGAAT GRAATTCC

CMCW41 DROSHA 3258C>T (R967W)

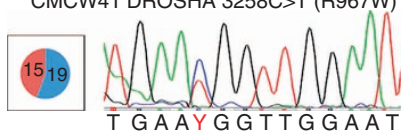

CMCW59 DICER1 5426G>T (G1809V)
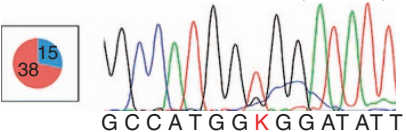

b

\begin{tabular}{|c|c|c|c|c|c|c|c|c|}
\hline Sample ID & DROSHA & DICER1 & MYCN & $A R I D 1 A$ & SMARCA4 & TP53 & $W T 1$ & CTNNB1 \\
\hline CMCW7 & D1151Y & $\mathrm{N}$ & $\mathrm{N}$ & $\mathrm{N}$ & $\mathrm{N}$ & $\mathrm{N}$ & $\mathrm{N}$ & $\mathrm{N}$ \\
\hline CMCW15 & E1147K & $\mathrm{N}$ & $\mathrm{N}$ & $\mathrm{N}$ & $\mathrm{N}$ & $\mathrm{N}$ & $\mathrm{N}$ & $\mathrm{N}$ \\
\hline CMCW79 & E1147K & $\mathrm{N}$ & $\mathrm{N}$ & $\mathrm{N}$ & $\mathrm{N}$ & $\mathrm{N}$ & $\mathrm{N}$ & $\mathrm{N}$ \\
\hline CMCW41 & $\mathrm{R} 967 \mathrm{~W}^{\#}$ & $\mathrm{~N}$ & $\mathrm{~N}$ & N2003S ${ }^{\S}$ & $\mathrm{N}$ & $\mathrm{N}$ & $\mathrm{N}$ & $\mathrm{N}$ \\
\hline CMCW39 & L728V & $\mathrm{N}$ & $\mathrm{N}$ & $\mathrm{N}$ & $\mathrm{N}$ & $\mathrm{N}$ & $\mathrm{N}$ & $\mathrm{N}$ \\
\hline CMCW53 & M120V" & $\mathrm{N}$ & $\mathrm{N}$ & $\mathrm{N}$ & $\mathrm{N}$ & $\mathrm{N}$ & $\mathrm{N}$ & $\mathrm{N}$ \\
\hline CMCW11 & $\mathrm{N}$ & $\begin{array}{l}\text { G1809, } \\
\text { |1102fsdel }\end{array}$ & $\mathrm{N}$ & $\mathrm{N}$ & $\mathrm{N}$ & V274L & $\mathrm{N}$ & $\mathrm{N}$ \\
\hline CMCW59 & $\mathrm{N}$ & G1809V & $\mathrm{N}$ & $\mathrm{N}$ & $\mathrm{N}$ & R342P & $\mathrm{N}$ & $\mathrm{N}$ \\
\hline CMCW23 & $\mathrm{N}$ & $\mathrm{N}$ & P44L & $\mathrm{N}$ & $\mathrm{N}$ & $\mathrm{R} 158 \mathrm{H}$ & $\mathrm{N}$ & $\mathrm{N}$ \\
\hline CMCW45 & $\mathrm{N}$ & $\mathrm{N}$ & P44L & $\mathrm{N}$ & $\mathrm{N}$ & $\mathrm{N}$ & $\mathrm{N}$ & $\mathrm{N}$ \\
\hline CMCW19 & $\mathrm{N}$ & $\mathrm{N}$ & $\mathrm{N}$ & L1871H & $\mathrm{N}$ & $\mathrm{N}$ & $\mathrm{N}$ & $\mathrm{N}$ \\
\hline CMCW21 & $\mathrm{N}$ & $\mathrm{N}$ & $\mathrm{N}$ & $\mathrm{N}$ & R469W & $\mathrm{N}$ & $\mathrm{N}$ & $\mathrm{N}$ \\
\hline CMCW69 & $\mathrm{N}$ & $\mathrm{N}$ & $\mathrm{N}$ & $\mathrm{N}$ & A747T & $\mathrm{N}$ & Q187* & $\mathrm{N}$ \\
\hline CMCW57 & $\mathrm{N}$ & $\mathrm{N}$ & $\mathrm{N}$ & $\mathrm{N}$ & $\mathrm{N}$ & $\mathrm{N}$ & V150।ई & $\mathrm{N}$ \\
\hline CMCW87 & $\mathrm{N}$ & $\mathrm{N}$ & $\mathrm{N}$ & $\mathrm{N}$ & $\mathrm{N}$ & $\mathrm{N}$ & $\mathrm{Y} 125^{*}$ & $\begin{array}{l}\text { S37C, } \\
\text { L160P }\end{array}$ \\
\hline CMCW51 & $\mathrm{N}$ & $\mathrm{N}$ & $\mathrm{N}$ & $\mathrm{N}$ & $\mathrm{N}$ & $N$ & Y192*§ & $\mathrm{N}$ \\
\hline CMCW17 & $\mathrm{N}$ & $\mathrm{N}$ & $\mathrm{N}$ & $\mathrm{N}$ & $\mathrm{N}$ & $\mathrm{N}$ & $N$ & T41A \\
\hline CMCW81 & $\mathrm{N}$ & $\mathrm{N}$ & $\mathrm{N}$ & $\mathrm{N}$ & $\mathrm{N}$ & $\mathrm{N}$ & $\mathrm{N}$ & 44 45del \\
\hline CMCW71 & $\mathrm{N}$ & $\mathrm{N}$ & $\mathrm{N}$ & $\mathrm{N}$ & $\mathrm{N}$ & $\mathrm{N}$ & $N$ & S45Y \\
\hline CMCW1 & $\mathrm{N}$ & $\mathrm{N}$ & $\mathrm{N}$ & $\mathrm{N}$ & $N$ & $\mathrm{~N}$ & $\mathrm{~N}$ & $\mathrm{~N}$ \\
\hline CMCW9 & $\mathrm{N}$ & $\mathrm{N}$ & $\mathrm{N}$ & $\mathrm{N}$ & $\mathrm{N}$ & $\mathrm{N}$ & $\mathrm{N}$ & $\mathrm{N}$ \\
\hline CMCW13 & $\mathrm{N}$ & $\mathrm{N}$ & $\mathrm{N}$ & $\mathrm{N}$ & $\mathrm{N}$ & $\mathrm{N}$ & $\mathrm{N}$ & $\mathrm{N}$ \\
\hline CMCW27 & $\mathrm{N}$ & $\mathrm{N}$ & $\mathrm{N}$ & $\mathrm{N}$ & $\mathrm{N}$ & $\mathrm{N}$ & $\mathrm{N}$ & $\mathrm{N}$ \\
\hline CMCW47 & $\mathrm{N}$ & $N$ & $\mathrm{~N}$ & $\mathrm{~N}$ & $\mathrm{~N}$ & $\mathrm{~N}$ & $\mathrm{~N}$ & $\mathrm{~N}$ \\
\hline 20 tumors & $\mathrm{N}$ & $\mathrm{N}$ & $\mathrm{N}$ & $\mathrm{N}$ & $\mathrm{N}$ & $\mathrm{N}$ & $\mathrm{N}$ & $\mathrm{N}$ \\
\hline
\end{tabular}

e

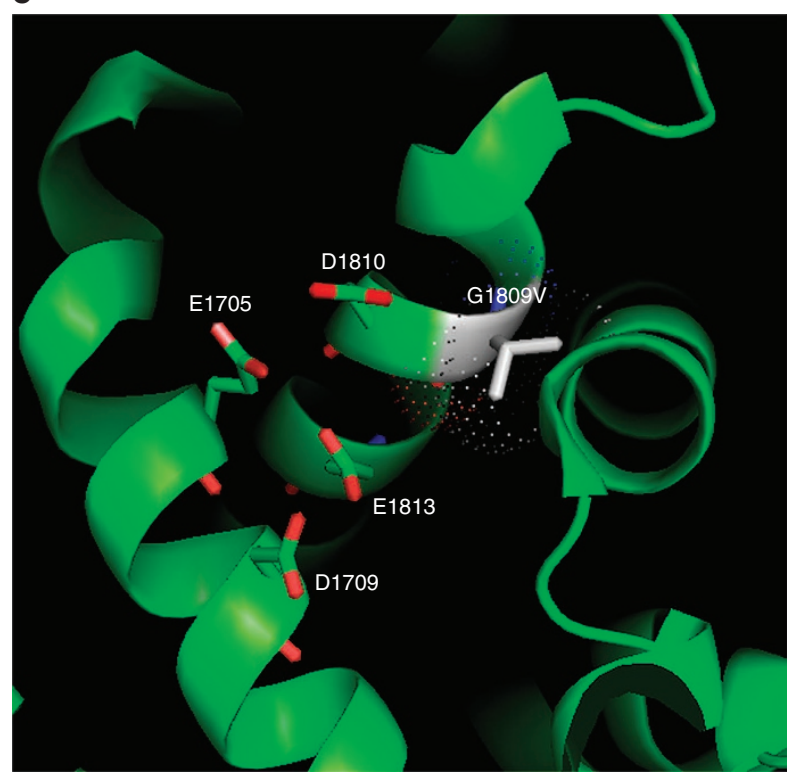

Figure 1 | Mutations in the miRNA biogenesis pathway in Wilms tumours. (a) Summary of somatic mutations detected by exome capture and massively parallel sequencing of matched Wilms tumours and germline DNA in the discovery set. (b) Mutations in miRNA biogenesis components are mutually exclusive with WT1 and CTNNB1 mutations. (c) Domain structure of DICER1 and DROSHA, indicating positions of mutations. Metal-binding residues in the RNase III domains are indicated in red. PAZ, Piwi-Argonaute-Zwille domain; DRBM, double-stranded RNA-binding motif. (d) Electropherograms from resequencing of selected mutations. Pie charts depict read counts from Illumina sequencing for reference (blue) and variant (red) alleles. (e) Modelling of G1809V mutation (silver) in the DICER1 metal-binding site, based on the structure of G. lamblia RNase III (PDB 2QVW). Numbering of equivalent Homo sapiens DICER1 residues is indicated. Dots indicate solvent-accessible surface.

metal-binding residues E969 and D973 in the RNase IIIA domain and $\mathrm{M} 120 \mathrm{~V}$ that is in the amino-terminal proline-rich domain (Fig. 1c). As germline DICER1 mutations are associated with elevated cancer susceptibility, we also tested these variants in vitro. The M120V mutation has no detectable effect on DROSHA activity, while the R967W mutation measurably reduces DROSHA activity in a substrate-specific manner (Fig. 2c). Thus, although the significance of the R967W variant is presently unclear, it is possible that analogous to germline DICER 1 mutations, this allele may confer an elevated inherited risk of Wilms tumour. None of the DROSHA mutations appeared to preferentially affect processing of $5 \mathrm{p}$ or $3 \mathrm{p}$ miRNAs.
We also tested the effects of the DGCR8 and TARBP2 mutations in vitro (Supplementary Fig. 2). DGCR8 S702N shows no impairment of DROSHA interaction or miRNA processing activity. Similarly, TARBP2 D221G is equivalent to wild type in its DICER1 interactions and processing activity. Therefore, although we cannot rule out an effect of these mutations in vivo, they do not appear to detectably affect activity in vitro. Of note, in addition to mutations in DROSHA, Torrezan et al. ${ }^{15}$ also identified non-synonymous missense mutations in DGCR8 and TARBP2, as well as XPO5 and DICER1; however, the functional impact of these mutations was not tested. 

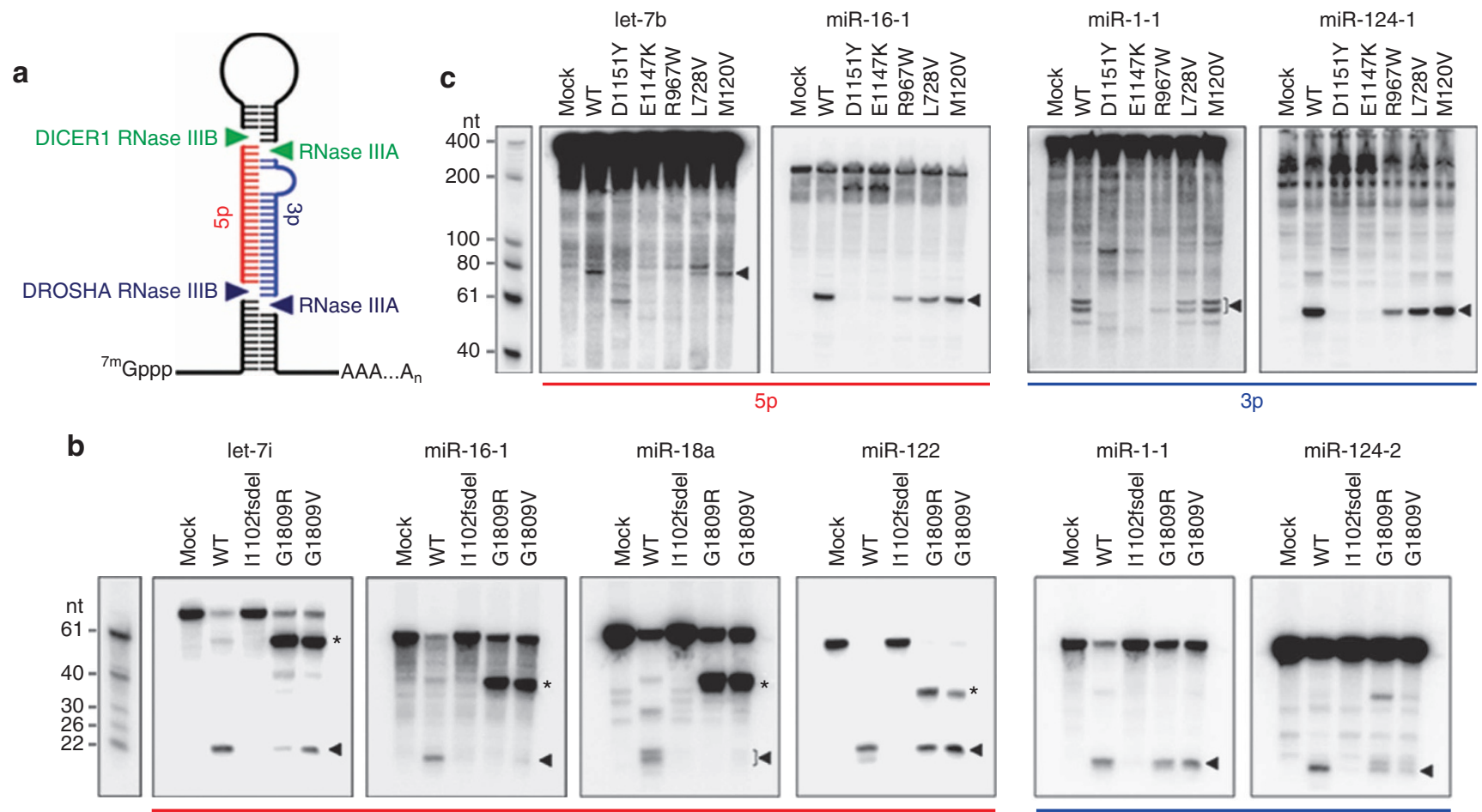

$5 p$

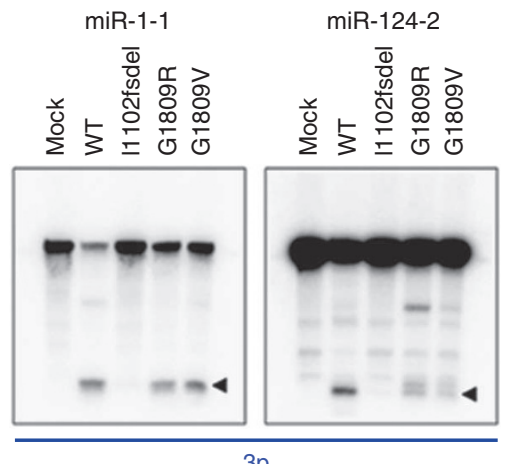

Figure 2 | Wilms tumour DICER1 and DROSHA mutations impair pri- and pre-miRNA processing in vitro. (a) Schematic of pri-miRNA indicating RNase III cleavage sites. (b) DICER1 mutations affect pre-miRNA processing. Wild-type or mutant DICER1 was purified by immunoprecipitation and tested against pre-miRNA substrates. Arrowhead, mature miRNA; asterisk, processing intermediate formed by DICER1 RIIIA cleavage in the absence of RIIIB activity. (c) Mutations in the RNase III domains impair DROSHA activity. Wild-type or mutant DROSHA was purified by immunoprecipitation and tested against pri-miRNA substrates. Arrowhead, processed pre-miRNA.

miRNA expression in DICER1- and DROSHA-mutant tumours. We next determined the effect of DICER1 and DROSHA mutations on miRNA expression in tumours. Small RNAs were prepared from Wilms tumours with and without DROSHA and DICER1 mutations, and analysed using highthroughput sequencing and quantitative PCR. Although both DICER1- and DROSHA-mutant tumours remain competent to produce some mature miRNAs, both tumour classes exhibit defective production of specific miRNAs (Fig. 3a). To gain insight into the possible oncogenic mechanisms of these mutations, we examined miRNAs that were expressed at lower levels in both DICER1- and DROSHA-mutant Wilms tumours (Fig. 3a). Included in this list are many members of the let-7 family, a group of highly evolutionarily conserved $5 p$ miRNAs that play roles in cell proliferation and differentiation, and have been implicated as tumour suppressors in human cancers ${ }^{35}$. By quantitative reverse transcription-PCR, we confirmed the lower expression of let-7 family members and several other miRNAs in all mutant and a few wild-type tumours (Fig. 3b). Therefore, our results show that mutations in DICER1 or DROSHA affect the biogenesis of selected miRNAs in tumours, and suggest that partially defective miRNA processing may drive the development of a subset of Wilms tumours via decreased expression of tumour suppressor miRNAs, including members of the let-7 family.

As predicted by the previous analyses of DICER1 RNase IIIBdomain mutations in cell lines ${ }^{28}$ and our in vitro processing experiments (Fig. 2a), DICER1 mutant tumours showed preferential impairment of expression of mature miRNAs derived from the $5^{\prime}$-arm of pre-miRNA hairpins compared with those derived from the $3^{\prime}$-arm (Fig. 3c,d). Out of 122 miRNAs expressed at lower levels in DICER1-mutant tumours compared with tumours with wild-type DICER1 and DROSHA ( $\log _{2}$ fold- change $<-0.5), 101$ derive from the $5^{\prime}$-arm, whereas 21 derive from the $3^{\prime}$-arm. One of the two tumours with a G1809 DICER1 mutation also harboured a germline frameshift deletion of the other allele. Notably, although this tumour (CMCW11) exhibited the most severe depletion of $5 \mathrm{p}$ miRNAs in our cohort, the tumour continued to express mature miRNAs (Fig. 3d), indicating that the G1809R mutant allele retained some capacity to produce miRNAs. A similar $3 p$ skewing of miRNA expression was recently reported in pleuropulmonary blastomas occurring in patients with germline-null and somatic missense mutations in DICER1 (refs 36,37).

DROSHA RNase IIIB mutations dominantly impair miRNA biogenesis. DROSHA acts at the pri-miRNA processing step, and, as expected, DROSHA mutant tumours show no $5 \mathrm{p} / 3 \mathrm{p}$ skewing (Fig. 3c,d). In conjunction with our in vitro processing assays, which showed DROSHA RIIIB mutants to be functional nulls, this led us to question why heterozygous DROSHA mutations at precise metal-binding residues appear to be selected for in Wilms tumours, rather than other types of heterozygous null mutations such as frameshifts, as has been observed in DICER1 in cancer predisposition syndromes ${ }^{5,32}$. We confirmed that DROSHA mutations in Wilms tumours were heterozygous and that both alleles were expressed (Supplementary Fig. 3). We thus hypothesized that these mutations, rather than behaving as simple loss-of-function alleles, behave in a dominant-negative manner to impair the function of the residual wild-type allele.

To directly test whether DROSHA RNase IIIB mutations function through a haploinsufficient or a dominant-negative mechanism, we used genome editing with transcription activatorlike effector nucleases (TALENs) to model DROSHA ${ }^{+/-}$and 

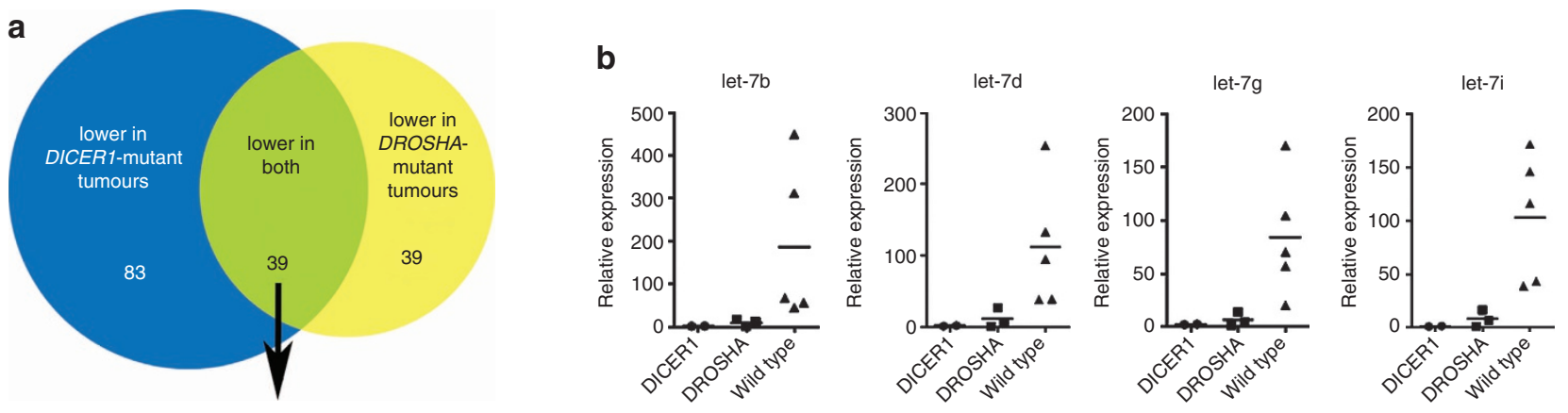

\begin{tabular}{lll}
\hline let-7b-5p & miR-146a-5p & miR-326 \\
let-7d-5p & miR-146b-5p & miR-328 \\
let-7g-5p & miR-151a-5p & miR-361-5p \\
let-7i-5p & miR-181b-5p & miR-376a-5p \\
miR-100-5p & miR-192-5p & miR-424-5p \\
miR-106b-5p & miR-195-5p & miR-497-3p \\
miR-127-5p & miR-204-5p & miR-497-5p \\
miR-134 & miR-214-5p & miR-504 \\
miR-135b-5p & miR-26b-5p & miR-505-3p \\
miR-136-5p & miR-28-3p & miR-542-5p \\
miR-139-5p & miR-299-5p & miR-574-5p \\
miR-140-3p & miR-29c-3p & miR-582-5p \\
miR-140-5p & miR-32-5p & miR-628-5p
\end{tabular}
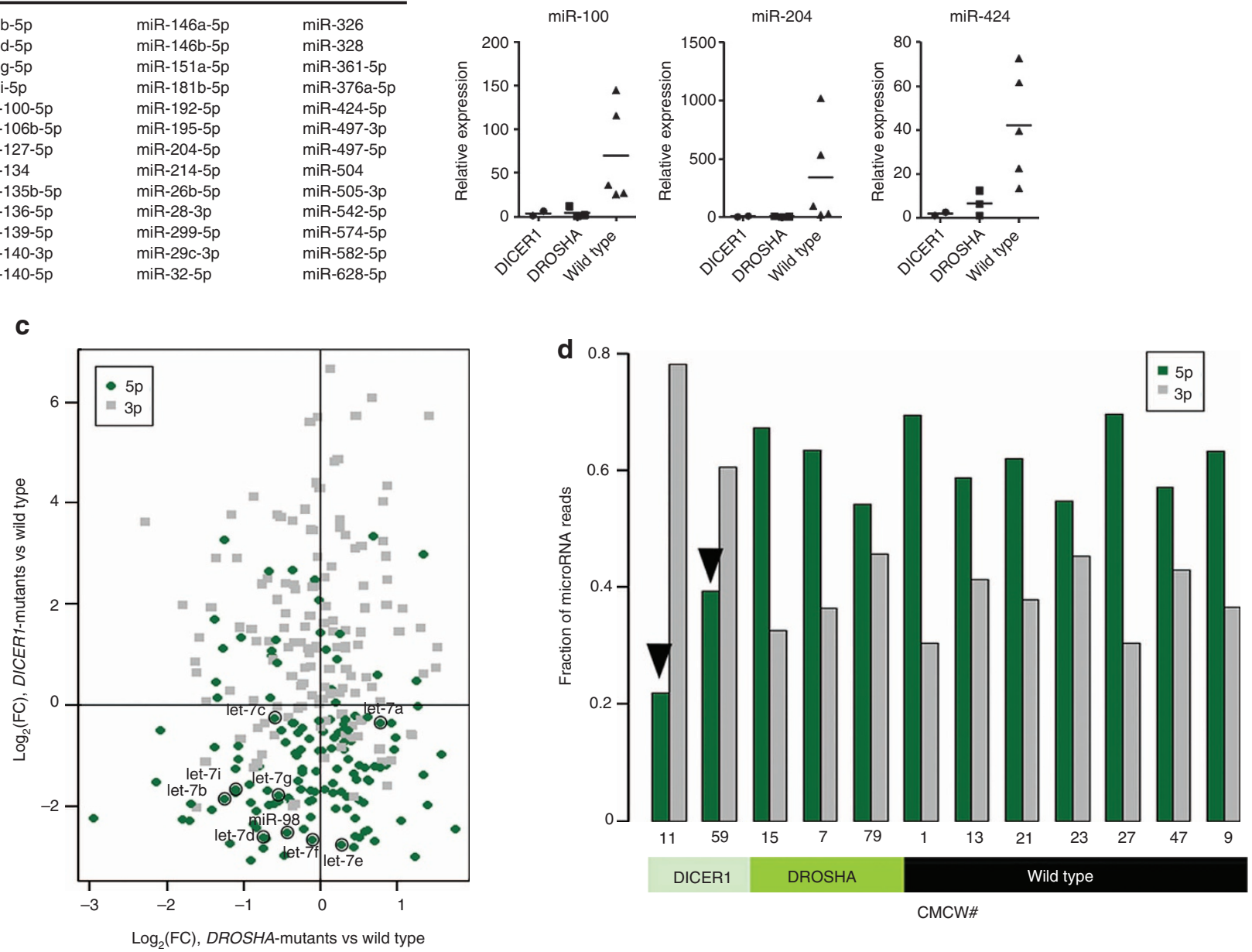

Figure 3 | DICER1 and DROSHA mutations impair miRNA biogenesis in Wilms tumours. (a) Summary of differentially expressed miRNAs in DICER1 and DROSHA-mutant tumours as determined by small RNA-seq. The 39 miRNAs whose expression is lower in both DICER1 and DROSHA-mutant tumours are listed. (b) Quantitative reverse transcription-PCR validation of expression of selected miRNAs in tumours with or without mutations in DROSHA and DICER1. (c) Fold-change in expression of $5^{\prime}$ (green) and 3' (grey)-derived miRNAs comparing DROSHA or DICER1 mutant tumours with non-mutant tumours. Members of the let-7 family are indicated. (d) Fraction of total miRNA reads deriving from the $5^{\prime}$ (green) or $3^{\prime}$ (grey) arm of the pre-miRNA hairpin for each tumour. Arrowheads indicate lower representation of $5^{\prime}$ miRNAs in DICER1 mutants.

DROSHA ${ }^{+/ E 1147 \mathrm{~K}}$ states in cultured human cells. In the absence of available genomically stable Wilms tumour cell lines, we engineered the DROSHA mutations into HCT116 cells, a wellcharacterized diploid human cancer cell line. Crucially, we introduced mutations into the endogenous DROSHA locus, ensuring physiologic expression levels and allowing us to directly test the haploinsufficiency versus dominant-negative models. Three independent clones harbouring distinct heterozygous frameshift mutations in the second coding exon and three independent clones harbouring the E1147K mutation were generated (Fig. 4a and Supplementary Fig. 4). Immunoblottings of the engineered cells confirmed that heterozygous null cells $(+/-)$ exhibit reduced DROSHA protein levels, while the E1147K mutation $(+/ \mathrm{EK})$ does not affect DROSHA expression (Fig. $4 \mathrm{~b}$ and Supplementary Fig. 4c,d). miRNA profiling of parental cells and each of the six mutant clones using Taqman arrays was then used to determine the global effects of DROSHA mutations on miRNA processing. Although both $D R O S H A^{+/-}$ and DROSHA $+/ \mathrm{EK}$ cells produced less mature miRNA overall compared with parental HCT116 cells, the E1147K mutation resulted in a significantly greater magnitude of miRNA repression (average $\log _{2}$ fold change -0.86 for $+/$ - versus -2.01 for $+/$ EK; Fig. 4c). When individual miRNAs were evaluated, no individual miRNAs were expressed at higher levels in either 
a
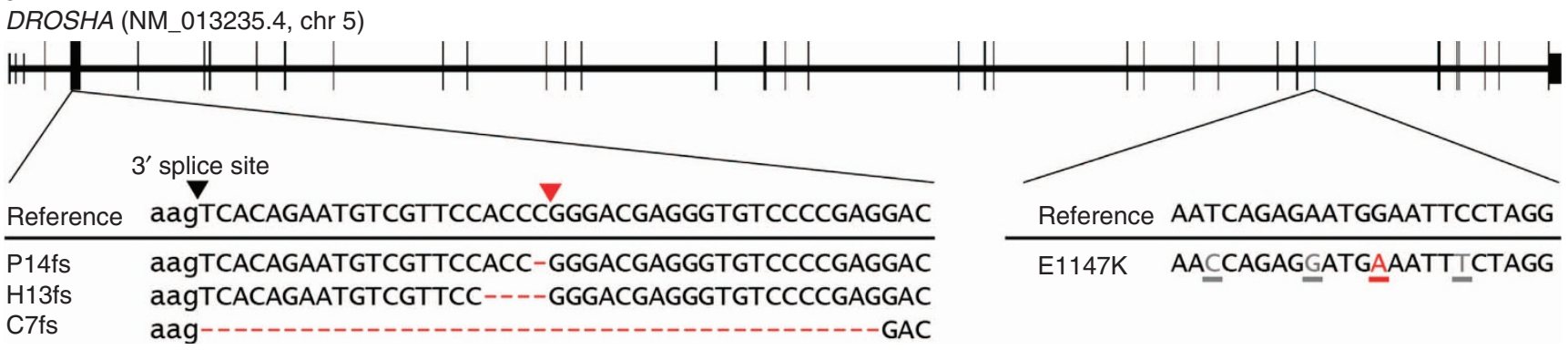

Reference AATCAGAGAATGGAATTCCTAGG

E1147K AACCAGAGGATGAAATTICTAGG

b
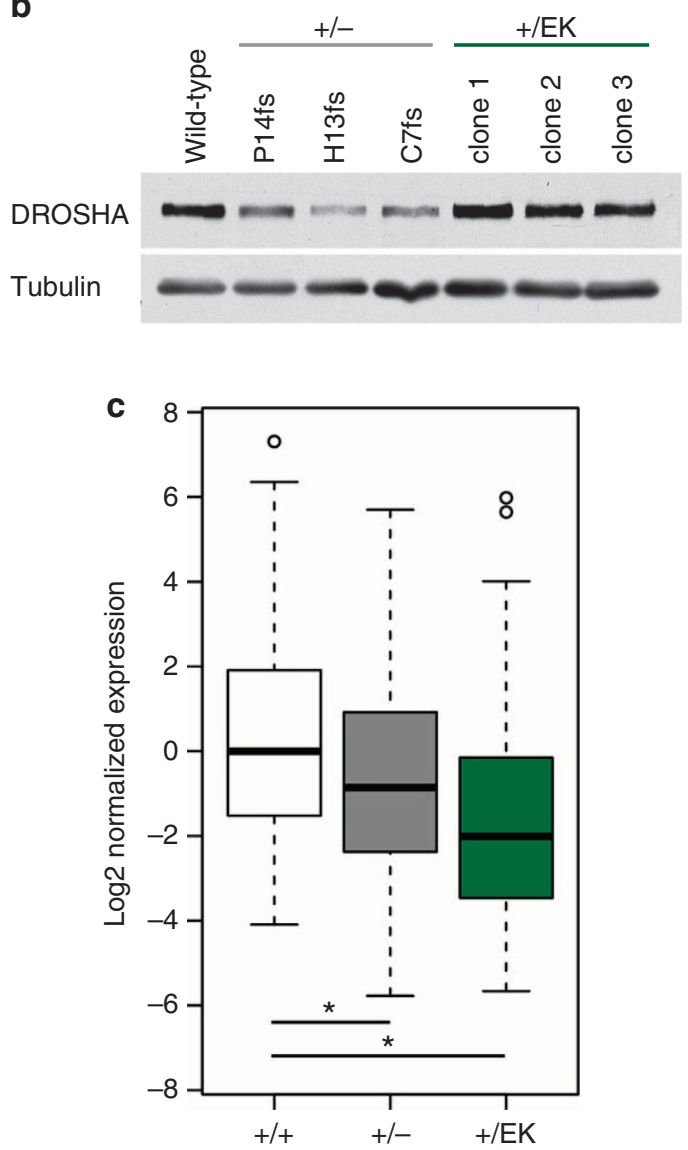

d
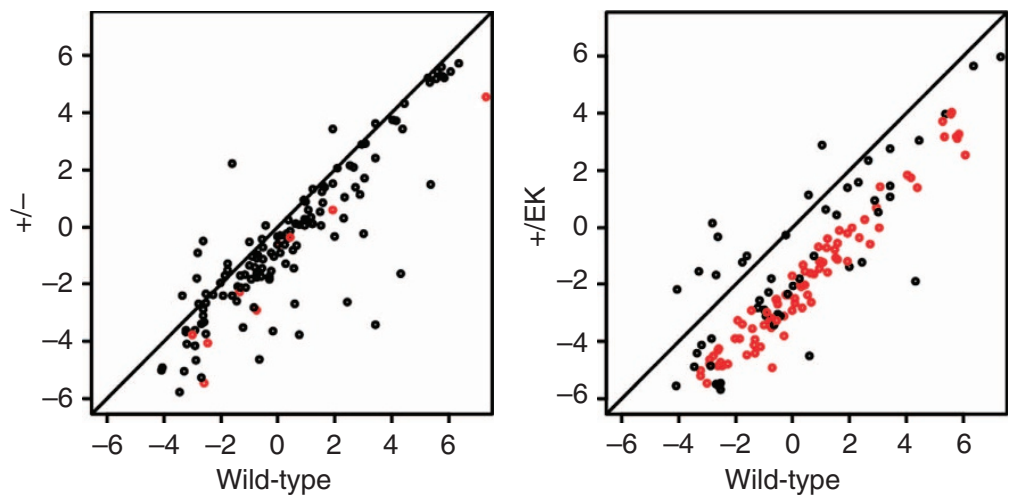

e

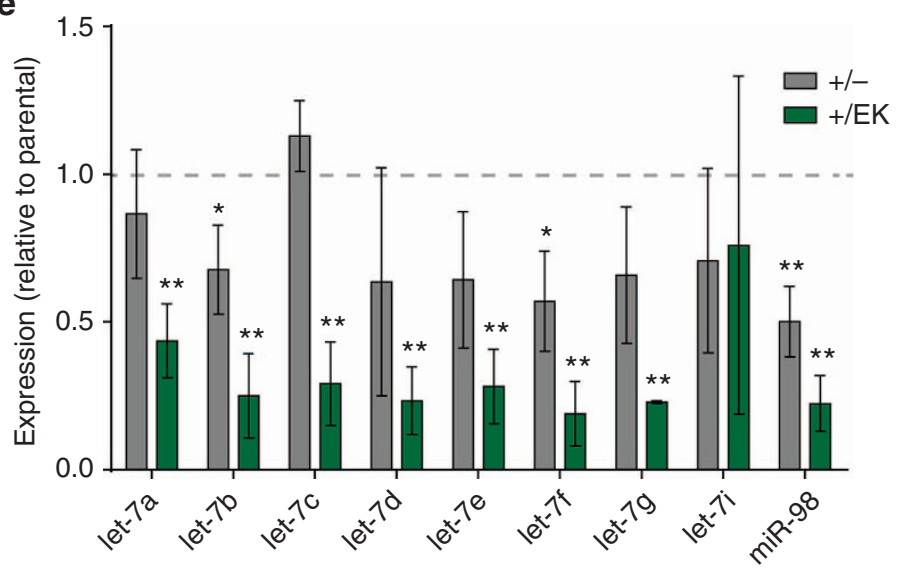

Figure 4 | The heterozygous DROSHA E1147K mutation acts as a dominant-negative allele. (a) TALEN-induced frameshift mutations in DROSHA+/cells (left) and knock-in mutations in DROSHA+/E1147K cells (right) are shown. Silent mutations, incorporated to prevent re-cleavage by TALENs, are denoted by grey underlined letters, while the non-synonymous mutation is in red. Intronic sequences in lower case. (b) Immunoblotting of DROSHA levels in parental and mutant cell lines. The full blot is shown in Supplementary Fig. 4. (c) Distribution of miRNA expression levels, displayed as log 2 (normalized expression), calculated by subtracting average $\Delta \mathrm{Ct}$ of each miRNA ( $\mathrm{Ct}_{\text {miRNA }}-\mathrm{Ct}_{\mathrm{UG}}$, averaged within each genotype) from the median parental $\Delta \mathrm{Ct}$ value. Distributions compared by paired two-tailed $t$-test; ${ }^{*} P<10^{-10}$. (d) Scatter plot of $\log _{2}$ (normalized expression) of individual miRNAs in DROSHA ${ }^{+} /-$and DROSHA $+/$ E1147K cells versus parental cells. Red dots represent miRNAs whose expression differed significantly $(P<0.05)$ between mutant cells and wild type; $P$-values computed by two-tailed t-test. (e) Expression of let-7 family members in DROSHA-mutant cells, relative to parental cells. Individual TaqMan assays for each miRNA were performed in triplicate for each of three clones within each genotype. Error bars represent mean \pm s.d. across three clones within each genotype. Dashed line represents expression in parental cells; $P$-values calculated by comparing expression in mutant versus parental cells by $z$-test. ${ }^{\star} P<0.05 ;{ }^{\star \star} P<0.005$.

mutant genotype, and only a few (9 out of 139) were expressed at statistically significantly lower levels in $D R O S H A^{+/}$cells compared with parental HCT116 cells (Fig. 4d and Supplementary Fig. 5a). In contrast, DROSHA ${ }^{+/ E 1147 \mathrm{~K}}$ cells showed statistically significantly impaired expression of a large majority of detectable miRNAs (85 out of 139; Fig. $4 \mathrm{~d}$ and Supplementary Fig. 5b). In keeping with our in vitro processing results (Fig. 2b) and analysis of miRNAs in Wilms tumours
(Fig. 3c,d), the E1147K mutation did not result in 5p/3p skewing of miRNA expression (Supplementary Fig. 5b). The effect of the EK mutation was particularly striking when considering the expression of let-7 miRNAs. Although some let-7 family members were modestly reduced in DROSHA ${ }^{+/-}$cells, production of eight out of nine assayed let-7 family members was dramatically impaired in DROSHA ${ }^{+/ E K}$ cells (Fig. 4e). Taken together, these data provide strong evidence that DROSHA RIIIB hotspot mutations act in a 
dominant-negative manner to impair processing of a large set of miRNAs that includes the let-7 family members.

\section{Discussion}

In this report, we demonstrate that widespread dysregulation of miRNA biogenesis is a key driving mechanism in Wilms tumour formation. We used exome sequencing of germline and tumour DNA to identify novel oncogenic mechanisms in Wilms tumour. Somatic and inherited mutations in key components of the miRNA biogenesis pathway, most prominently in the RNase IIIB (RIIIB) domains of DICER1 and DROSHA, are recurrent events in Wilms tumour. So-called DICER1 'hotspot' mutations prevent processing of $5 p$-derived miRNAs, which reverses the $5 \mathrm{p} / 3 \mathrm{p}$ bias typically seen in Wilms tumour miRNA expression. RIIIBmutant DROSHA, on the other hand, is completely unable to process pri-miRNAs for both $5 \mathrm{p}$ - and $3 \mathrm{p}$-derived miRNAs, and even the germline RIIIA mutation, R967W, demonstrated a partial loss of function. To precisely model the effect of missense mutations in tumours, we used genome engineering to show that a heterozygous DROSHA RIIIB mutation is more deleterious than simply a heterozygous null allele, confirming the hypothesis that these mutations impair global miRNA biogenesis through a dominant-negative mechanism. Most importantly, mutations in either DICER1 or DROSHA result in the loss of key tumour suppressor miRNAs, including the let-7 family as a whole.

Our data thus provide a mechanistic understanding of the selective pressures that give rise to the observed mutation spectra of DICER1 and DROSHA in Wilms tumours. Prior work has established that in mouse models of lung adenocarcinoma, softtissue sarcoma and retinoblastoma, heterozygous Dicer1 deletion accelerates tumorigenesis, whereas complete Dicer1 loss-offunction is strongly selected against ${ }^{38,39}$. Moreover, loss of the residual wild-type allele in human tumours harbouring germline or somatic DICER1 mutations is rarely observed ${ }^{12,32,40,41}$. These data indicate that in specific cancer settings, partial impairment of miRNA biogenesis is advantageous, yet residual activity of the miRNA pathway is needed for cell viability and/or other aspects of tumour biology. Importantly, this is probably not the case in all tumour types, as complete DICER1 loss-of-function was observed in a case of pineoblastoma ${ }^{42}$. Nevertheless, our finding of partial, but never complete, loss of miRNA processing through DROSHA and DICER1 mutations in Wilms tumours suggest that residual miRNA activity is required in this cancer. Furthermore, it is reasonable to speculate that somatic heterozygous DICER1 RNase IIIB domain mutations, especially when they occur in a patient with a germline DICER1-null allele, provide the optimal balance of impaired production of tumour suppressor miRNAs (many of which derive from the $5^{\prime}$-arm of miRNA hairpins such as members of the let-7 family), while allowing sufficient biogenesis of miRNAs that are required for tumour viability. In the case of DROSHA, heterozygous null mutations have very little impact on miRNA production, as revealed by our studies in genetically engineered cell lines, and are thus not observed in tumours. Heterozygous DROSHA RNase IIIB mutations, in contrast, have a dominant-negative effect on miRNA production, impairing production of the let-7 family and numerous other miRNAs, yet apparently allowing sufficient residual miRNA processing activity to support tumour growth. Finally, it is important to note that functions beyond miRNA processing have been ascribed to both DROSHA and DICER1, such as participation in the DNA damage response ${ }^{43}$, warranting further investigation of these non-canonical functions in Wilms tumour pathogenesis.

As shown in Fig. 3b, some Wilms tumours without identifiable mutations in the miRNA processing machinery also show moderately reduced levels of let-7 family members, suggesting that other mechanisms of reducing let-7 levels may be active in these tumours. Upregulation of LIN28A or LIN28B is known to suppress biogenesis of the let-7 family in various cancers ${ }^{44-46}$, including Wilms tumour ${ }^{44}$, and Myc induces LIN28B expression to repress let-7 (ref. 47). Urbach et al. ${ }^{48}$ recently showed that conditional expression of LIN28 in developing mouse kidney mesodermal precursor cells is sufficient to cause Wilms tumours, an effect which could be suppressed by expression of a let-7 family member. In our tumour cohort, three of nine tumours examined expressed relatively high levels of LIN28A and/or LIN28B (Supplementary Fig. 6), consistent with the reported prevalence of LIN28 overexpression in Wilms tumours ${ }^{44}$. However, there was no direct correlation between LIN28 expression and downregulation of mature let-7 family members, suggesting that additional mechanisms, beyond DROSHA and DICER 1 mutations and LIN28 overexpression, may lead to let-7 repression in some tumours. Taken together, these data indicate that loss of let-7 expression, whether by mutation of miRNA processing genes or by other mechanisms, may represent a central mechanism of oncogenesis in Wilms tumours.

\section{Methods}

Exome sequencing and analysis. The tumour specimens used in the study were residual tissue from the patients' surgical resection specimens and stored either with informed consent in the Pediatric Biospecimen Repository or in the Department of Pathology archives at Children's Medical Center. This study was performed under a protocol approved by the Institutional Review Board of the University of Texas Southwestern Medical Center. Indexed DNA libraries were constructed per the TruSeq Exome Enrichment Kit, as per the provided protocol Target enrichment was then performed by pooling four indexed samples together and using the human TruSeq Exome Enrichment kit for each pool, also as per protocol. This kit targets $62 \mathrm{Mb}$ of exomic sequences in the human genome, including $5^{\prime}$-untranslated region, $3^{\prime}$-untranslated region, miRNA and other noncoding RNA regions. The resulting libraries were sequenced using the HiSeq 2000, producing $100 \mathrm{bp}$ paired-end reads.

Reads were demultiplexed using casava version 1.8.2, allowing up to one mismatch in the index sequence and clusters not passing filter were excluded from the resulting fastq files. The reads were then quality-checked using fastQC (0.8.0). Each sample's reads were aligned to the hg19 build of the human genome reference sequence using BWA (v 0.6.2), and duplicates were removed using Picard's MarkDuplicates (1.41). Sample level realignment around indels and base quality recalibration were performed using GATK 1.4.6 (Broad Institute, Boston, MA) (http://www.broadinstitute.org/gatk/guide/topic?name=best-practices). Coverage was also calculated using GATK, and an average coverage of $52 \times$ was achieved across targeted regions, with $91 \%$ of bases covered at $>10 \times$ and $85 \%$ of bases covered at $>20 \times$. Single-nucleotide polymorphisms (SNPs) and Indels in the targeted regions were called using GATK's UnifiedGenotyper, followed by hard filtering (indels) and VariantRecalibration (SNPs). During recalibration, data from the OMNI and HapMap SNP chips were used as training sets. Those variants passing filters were annotated using successive ANNOVAR runs (2012-05-25), wherein dbSNP IDs, 1000 Genomes frequencies, polyPhen and SIFT scores were added. ANNOVAR also provided genomic location, and when variants were found to be exonic, also annotated the gene name, transcript ID, functional change, nucleotide and amino acid change of each variant.

We generated a median of 88 million raw reads per tumour sample. In the discovery set, deviations from the reference genome were identified in both tumour and matched germline samples. Candidate somatic mutations were identified if they were not present on any reads in the germline samples; seen on at least three reads in tumour samples; non-synonymous; not present in the dbSNP or 1000 Genomes databases; and covered at a minimum total read depth of $6 \times$. Mutations in DROSHA and DICER1 were validated by Sanger sequencing with primers designed using Primer-BLAST. Similar filters were used to uncover single nucleotide variants in the validation set.

Mutagenesis. The V5-DROSHA expression plasmid was constructed by subcloning DROSHA from pcDNA4/TO/cmycDrosha ${ }^{49}$ into pcDNA3.1/V5-HisTOPO (Invitrogen). The QuikChange Lightning Site-Directed Mutagenesis kit (Stratagene) was used to introduce mutations into plasmids encoding V5DROSHA, FLAG-DGCR8 (ref. 49), V5-DICER1 (ref. 50) and FLAG-TARBP2 (ref. 50). Primers used for mutagenesis are listed in Supplementary Data 4.

Cell Culture and immunoprecipitation. For DROSHA and DICER1 processing assays, HEK293T cells were seeded at a density of $5 \times 10^{6}$ cells per $100 \mathrm{~mm}$ plate $12 \mathrm{~h}$ before transfection. Cells were transfected with V5-DICER1 or a mixture of V5-DROSHA and FLAG-DGCR8 expression plasmids using X-tremeGENE 9 
(Roche Applied Science) following the manufacturer's protocol. Forty-eight hours after transfection, cells were washed with cold PBS and collected into $600 \mu \mathrm{l}$ of IP buffer containing $20 \mathrm{mM}$ Tris $\cdot \mathrm{HCl}$ (pH 8.0), $150 \mathrm{mM} \mathrm{NaCl}, 1 \mathrm{mM}$ EDTA, $0.5 \%$ Nonidet-40 and complete EDTA-free protease inhibitor (Roche Applied Science). Cells were lysed by sonication in a Bioruptor (Diagenode) and then centrifuged at $12,000 \mathrm{~g}$ for $10 \mathrm{~min}$ at $4^{\circ} \mathrm{C}$. The supernatant was collected and $15 \mu \mathrm{l}$ was saved as total input. The remaining lysate was incubated with $3 \mu \mathrm{g}$ of anti-V5 mouse monoclonal antibody (Invitrogen) for $30 \mathrm{~min}$ on ice. Thirty microlitres $(900 \mu \mathrm{g})$ of protein G Dynabeads (Invitrogen) were added and lysates were rotated for an additional $2 \mathrm{~h}$ at $4{ }^{\circ} \mathrm{C}$. The beads were washed five times in IP buffer and then twice in DROSHA/DICER1 assay buffer (20 mM Tris $\cdot \mathrm{HCl}(\mathrm{pH} 8.0), 100 \mathrm{mM} \mathrm{KCl}$, $0.2 \mathrm{mM}$ EDTA). Ninety per cent of the beads were resuspended in $50-70 \mu \mathrm{l}$ DROSHA/DICER1 assay buffer for enzymatic assays and the remaining beads were resuspended in Laemmli buffer for western blot analysis.

A similar protocol was followed for analysis of DGCR8 and TARBP2 mutations with the following modifications: V5-DROSHA was co-transfected with FlagDGCR8 wild type or S702N. V5-DICER1 was co-transfected with Flag-TARBP2 wild type or D221G. Complexes were immunoprecipitated with $6 \mu \mathrm{g}$ of anti-Flag M2 mouse monoclonal antibody (Sigma) and then resuspended in DROSHA/ DICER1 assay buffer for enzymatic assays or Laemmli buffer for western blots.

DROSHA and DICER1 processing assays. Pri-miRNA substrates consisting of miRNA hairpins and $\sim 80-150$ bp of genomic flanking sequence were amplified from human genomic DNA and either cloned into the NheI site of pcDNA3.1 (+) (miR-1-1 and miR-124-1) or used as in vitro transcription templates directly (let$7 \mathrm{~b}$ and miR-16-1, with T7 promoter in the $5^{\prime}$ primer). Primer sequences are provided in Supplementary Data 4. Non-radiolabelled pri-miRNAs were in vitro transcribed with XbaI-linearized plasmids (miR-1-1 and miR-124-1) or PCR fragments (let-7b and miR-16-1) using the MAXIscript kit (Ambion) and subsequentely gel purified. DROSHA processing assays were carried out by incubating $10 \mu \mathrm{l}$ of immunoprecipitated beads in DROSHA/DICER1 assay buffer prepared as described above, $2 \mu \mathrm{l}$ of $64 \mathrm{mM} \mathrm{MgCl} 2,0.2 \mu \mathrm{l}$ of pri-miRNA ( $\sim 0.3 \mathrm{pmol}), 0.6 \mu \mathrm{l}$ of RNaseOUT (Invitrogen) and $7.2 \mu \mathrm{l}$ of diethylpyrocarbonate-treated water at $37^{\circ} \mathrm{C}$ for $30 \mathrm{~min}$ and then stopped by chilling samples on ice. After adding $20 \mu \mathrm{l}$ of Gel Loading Buffer II (Ambion), reaction mixtures were boiled at $95^{\circ} \mathrm{C}$ for $3 \mathrm{~min}$ and then chilled on ice. Magnetic beads were removed and the supernatant was loaded onto a $15 \%$ denaturing polyacrylamide gel. Pre-miRNAs and processing intermediates were transferred onto nitrocellulose membranes in $0.5 \times$ TBE buffer at $250 \mathrm{~mA}$ for $2 \mathrm{~h}$. After ultraviolet cross-linking for $50 \mathrm{~mJ}$, membranes were incubated overnight at $42{ }^{\circ} \mathrm{C}$ with Ultrahyb Oligo Buffer (Ambion) containing ${ }^{32} \mathrm{P}$ endlabelled oligonucleotide probes perfectly complementary to the mature miRNA sequences. Membranes were then washed three times with Wash Buffer $(2 \times$ SSC, $0.5 \%$ SDS) at $42{ }^{\circ} \mathrm{C}$ and exposed to phosphor screens (GE Healthcare).

DICER1 processing assays were performed similarly using synthesized RNA oligos identical in sequence to human pre-miRNAs as substrates (sequences provided in Supplementary Data 4). Enzymatic mixtures consisted of $10 \mu \mathrm{l}$ of immunoprecipitated beads, $2 \mu \mathrm{l}$ of $20 \mathrm{mM} \mathrm{MgCl}, 0.2 \mu \mathrm{l}$ of $0.4 \mu \mathrm{M}$ pre-miRNA, $0.1 \mu \mathrm{l}$ of $100 \mathrm{mM}$ dithiothreitol, $0.5 \mu \mathrm{l}$ of RNaseOUT (Invitrogen) and $7.2 \mu \mathrm{l}$ of DEPC water.

Small RNA sequencing. Small RNAwas prepared from $50 \mathrm{ng}$ of each tumour sample using the mirVana PARIS kit according to standard manufacturer's protocol (Life Technologies). The small RNA libraries were prepared using the protocol for small RNA library preparation in the SOLiD Total RNA-Seq kit from Life Technologies. The resulting libraries were sequenced on the SOLiD 5500xl. The reads produced were $35 \mathrm{bp}$ single end. The machine demultiplexed the individual samples' reads on-machine. Demultiplexed reads from the machine were analysed using LifeScope (v. 2.5.1) using the default small RNA parameters for analysis. The reads were first mapped to a 'filter sequence' fasta file containing SOLiD adapter sequences, ribosomal RNA, transfer RNA and other 'junk' RNA sequences. The reads mapping to this file were excluded from further analysis. The remaining reads were then mapped to the hg19 build of the human genome and also to miRBase sequences. The algorithm used a seed-and-extend method for mapping reads, to better identify the ends of the miRNAs. The mapped reads were annotated according to both known precursor and mature miRNA sequences from miRBase. Using the information from the mapped bam files, coverage counts of mature and precursor sequences, as well as coverage wiggle files were produced. Read counts were categorized as being derived from the $5 \mathrm{p}$ or $3 \mathrm{p}$ arm according to their entries in http://www.mirbase.org. Next, the miRNAs that were detectable in all 12 tumours were analysed for differential expression using the DESeq v1.10.1 package of R/Bioconductor.

Quantitative PCR. Small RNAs were isolated from $50 \mathrm{ng}$ of each tumour sample as above. All samples were analysed using the RNA 6000 Nano LabChip (Agilent Technologies) on a 2100 Bioanalyzer (Agilent Technologies), and we defined a minimum RNA Integrity Number $>5$ to be suitable for further evaluation by TaqMan reverse transcription-PCR analysis ${ }^{51}$. Taqman miRNA assays (Invitrogen) for following target miRNAs were used to measure their expression in tumour samples: let-7b (Assay ID: 002619), let-7d (Assay ID: 002283), let-7g
(Assay ID: 002282), let-7i (Assay ID: 002221), miR-100 (Assay ID: 000437), miR204 (Assay ID: 000508) and miR-424 (Assay ID: 000604). The reference gene snU6 (Assay ID: 001973) was used for normalization. The RT ${ }^{2}$ qPCR Primer Assay (Qiagen) was used to measure LIN28A (Assay ID: PPH10338B) and LIN28B (Assay ID: PPH57843B).

Genetic engineering of cell lines. Pairs of TALENs were designed to target specific DROSHA sequences using ZiFit Targeter (zifit.partners.org) ${ }^{52}$. A pair of TALENs were designed to target a SmaI site in the second coding exon to create a null mutant through the introduction of indel mutations, while a second pair of TALENs were designed against the region surrounding codon 1147 to create the knock-in mutation through homologous recombination. TALENs were constructed using the Joung lab REAL assembly TALEN kit (www.addgene.org/ talengineering $)^{53}$. Complete amino acid sequences of TALENs are provided in Supplementary Methods. A homology template for the E1147K mutation was created by amplifying arms from genomic DNA (primers provided in Supplementary Table 4) and mutating $1 \mathrm{bp}$ to model the E1147K mutation; three more silent mutations were also introduced into the TALEN-binding site to prevent re-cleavage (mutations shown in Fig. 4a). Homology arms were ligated around a lox-stop-puro-lox cassette (from Addgene plasmid 11584 (ref. 54)), from which the stop elements had been excised.

The TALEN pair targeting the second coding exon was co-transfected into HCT116 cells with a green fluorescent protein-expressing plasmid using Fugene HD (Promega), and $48 \mathrm{~h}$ later the brightest $10 \%$ of cells were collected by FACS sorting. Colonies were expanded from single cells and genotyped for heterozygous destruction of the SmaI site and positive clones were confirmed by Sanger sequencing. Three heterozygous clones with independent frameshift mutations were expanded for further analysis.

The second TALEN pair was co-transfected with the homology template. Seventy-two hours after transfection, the cells were transferred to $1 \mu \mathrm{g} \mathrm{ml}^{-1}$ puromycin-containing media for 4 days and then seeded into 96-well plates for single-cell cloning. Puromycin-resistant colonies were genotyped by PCR, using a forward primer inside the puro cassette and a reverse primer outside the homology arm. The presence of the correctly targeted heterozygous mutations was confirmed by sequencing. Three correctly targeted clones were then transiently transfected with a Cre-expressing plasmid to remove the puro cassette and re-expanded from single cells to produce three independently derived clonal lines with the targeted allele.

Immunoblotting. Western blotting was performed using monoclonal antibodies against DROSHA (D28B1, Cell Signaling Technology, 1:1,000) and tubulin (clone DM1A, Sigma, 1:10,000)

Analysis of miRNA expression in engineered cell lines. miRNA expression was profiled in three independent heterozygous Drosha-null and E1147K cell lines and three biological replicates of parental HCT116 cells. Cells $\left(4 \times 10^{6}\right)$ were plated in $10-\mathrm{cm}$ dishes and total RNA was extracted the following day using the mirVana miRNA isolation kit (Life Technologies). Complementary DNA was reversetranscribed using the TaqMan MicroRNA Reverse Transcription Kit and Megaplex RT primers (Human Pool A v2.1), mixed with TaqMan Universal PCR Master Mix and loaded onto TaqMan Array Human MicroRNA A Cards 2.0 (Life Technologies). Following amplification on an Applied Biosystems 7900HT Real-Time PCR System, data were analysed using SDS software v2.4 and RQ manager v1.2.1 (Life Technologies). Raw Ct values were exported for statistical analysis.

miRNAs whose mean $C_{t}$ values were $>32$ in any of the three groups were discarded. Global miRNA expression was assayed by TaqMan array, and for each miRNA, $\Delta \mathrm{C}_{\mathrm{t}}$ was calculated by normalizing to $\mathrm{U} 6\left(\mathrm{Ct}_{\mathrm{miRNA}}-\mathrm{Ct}_{\mathrm{UG}}\right)$. Average $\Delta \mathrm{C}$ value for each miRNA was then calculated within each genotype and $\log 2$ of normalized expression was calculated by subtracting each average $\Delta C_{t}$ from the median parental $\Delta \mathrm{C}_{\mathrm{t}}$ value. Distributions were compared by paired $t$-test. Levels of let-7 family members were validated using individual TaqMan assays from Life Technologies.

\section{References}

1. Chu, A. et al. Wilms' tumour: a systematic review of risk factors and meta-analysis. Paediatr. Perinat. Epidemiol. 24, 449-469 (2010)

2. Rivera, M. N. \& Haber, D. A. Wilms' tumour: connecting tumorigenesis and organ development in the kidney. Nat. Rev. Cancer 5, 699-712 (2005).

3. Ko, E. Y. \& Ritchey, M. L. Current management of Wilms' tumor in children. J. Pediatr. Urol. 5, 56-65 (2009).

4. Kalapurakal, J. A. et al. Management of Wilms' tumour: current practice and future goals. Lancet Oncol. 5, 37-46 (2004).

5. Murphy, W. M., Grignon, D. J. \& Perlman, E. J. in AFIP Atlas of Tumor Pathology Series 4. Tumors of the Kidney, Bladder, and Related Urinary Structures (ed. Silverberg, S. G.) (American Registry of Pathology, 2004).

6. Robison, L. L. The Childhood Cancer Survivor Study: a resource for research of long-term outcomes among adult survivors of childhood cancer. Minn. Med. 88, 45-49 (2005) 
7. Wright, K. D., Green, D. M. \& Daw, N. C. Late effects of treatment for Wilms tumor. Pediatr. Hematol. Oncol. 26, 407-413 (2009).

8. Geenen, M. M. et al. Medical assessment of adverse health outcomes in longterm survivors of childhood cancer. JAMA 297, 2705-2715 (2007).

9. Sasso, G., Greco, N., Murino, P. \& Sasso, F. S. Late toxicity in Wilms tumor patients treated with radiotherapy at 15 years of median follow-up. J. Pediatr. Hematol. Oncol. 32, e264-e267 (2010).

10. Ruteshouser, E. C., Robinson, S. M. \& Huff, V. Wilms tumor genetics: mutations in WT1, WTX, and CTNNB1 account for only about one-third of tumors. Genes Chromosomes Cancer 47, 461-470 (2008).

11. Foulkes, W. D. et al. Extending the phenotypes associated with DICER1 mutations. Hum. Mutat. 32, 1381-1384 (2011).

12. Slade, I. et al. DICER1 syndrome: clarifying the diagnosis, clinical features and management implications of a pleiotropic tumour predisposition syndrome. J. Med. Genet. 48, 273-278 (2011).

13. Klein, S. et al. Expanding the phenotype of mutations in DICER1: mosaic missense mutations in the RNase IIIb domain of DICER1 cause GLOW syndrome. J. Med. Genet. 51, 294-302 (2014).

14. Wu, M. K. et al. Biallelic DICER1 mutations occur in Wilms tumours. J. Pathol. 230, 154-164 (2013)

15. Torrezan, G. T. et al. Recurrent somatic mutation in DROSHA induces microRNA profile changes in Wilms tumour. Nat. Commun. 5, 4039 (2014)

16. Lee, R. S. et al. A remarkably simple genome underlies highly malignant pediatric rhabdoid cancers. J. Clin. Invest. 122, 2983-2988 (2012).

17. Schwartzentruber, J. et al. Driver mutations in histone H3.3 and chromatin remodelling genes in paediatric glioblastoma. Nature 482, 226-231 (2012).

18. Hasselblatt, M. et al. Nonsense mutation and inactivation of SMARCA4 (BRG1) in an atypical teratoid/rhabdoid tumor showing retained SMARCB1 (INI1) expression. Am. J. Surg. Pathol. 35, 933-935 (2011).

19. Pugh, T. J. et al. Medulloblastoma exome sequencing uncovers subtype-specific somatic mutations. Nature 488, 106-110 (2012).

20. Robinson, G. et al. Novel mutations target distinct subgroups of medulloblastoma. Nature 488, 43-48 (2012).

21. Sausen, M. et al. Integrated genomic analyses identify ARID1A and ARID1B alterations in the childhood cancer neuroblastoma. Nat. Genet. 45, 12-17 (2013).

22. Pugh, T. J. et al. The genetic landscape of high-risk neuroblastoma. Nat. Genet. 45, 279-284 (2013).

23. Nisen, P. D., Zimmerman, K. A., Cotter, S. V., Gilbert, F. \& Alt, F. W. Enhanced expression of the N-myc gene in Wilms' tumors. Cancer Res. 46, 6217-6222 (1986).

24. Williams, R. D. et al. Molecular profiling reveals frequent gain of MYCN and anaplasia-specific loss of $4 \mathrm{q}$ and $14 \mathrm{q}$ in Wilms tumor. Genes Chromosome Cancer 50, 982-995 (2011).

25. Williams, R. D. et al. Subtype-specific FBXW7 mutation and MYCN copy number gain in Wilms' tumor. Clin. Cancer Res. 16, 2036-2045 (2010).

26. Krol, J., Loedige, I. \& Filipowicz, W. The widespread regulation of microRNA biogenesis, function and decay. Nat. Rev. Genet. 11, 597-610 (2010).

27. Lee, Y. et al. The nuclear RNase III Drosha initiates microRNA processing. Nature 425, 415-419 (2003).

28. Gurtan, A. M., Lu, V., Bhutkar, A. \& Sharp, P. A. In vivo structure-function analysis of human Dicer reveals directional processing of precursor miRNAs RNA 18, 1116-1122 (2012).

29. Han, J. et al. The Drosha-DGCR8 complex in primary microRNA processing Genes Dev. 18, 3016-3027 (2004).

30. Blaszczyk, J. et al. Crystallographic and modeling studies of RNase III suggest a mechanism for double-stranded RNA cleavage. Structure 9, 1225-1236 (2001).

31. Zhang, H., Kolb, F. A., Jaskiewicz, L., Westhof, E. \& Filipowicz, W. Single processing center models for human Dicer and bacterial RNase III. Cell 118, 57-68 (2004).

32. Heravi-Moussavi, A. et al. Recurrent somatic DICER1 mutations in nonepithelial ovarian cancers. New Engl. J. Med. 366, 234-242 (2012).

33. Anglesio, M. et al. Cancer-associated somatic DICER1 hotspot mutations cause defective miRNA processing and reverse strand expression bias to predominantly mature $3 \mathrm{p}$ strands through loss of $5 \mathrm{p}$ strand cleavage. J. Pathol. 229, 400-409 (2013).

34. Macrae, I. J. et al. Structural basis for double-stranded RNA processing by Dicer. Science 311, 195-198 (2006).

35. Bussing, I., Slack, F. J. \& Grosshans, H. let-7 microRNAs in development, stem cells and cancer. Trends Mol. Med. 14, 400-409 (2008).

36. Pugh, T. J. et al. Exome sequencing of pleuropulmonary blastoma reveals frequent biallelic loss of TP53 and two hits in DICER1 resulting in retention of $5 \mathrm{p}$-derived miRNA hairpin loop sequences. Oncogene doi:101038/onc.2014.150 (2014)

37. Seki, M. et al. Biallelic DICER1 Mutations in Sporadic Pleuropulmonary Blastoma. Cancer Res. 74, 2742-2749 (2014).

38. Kumar, M. S. et al. Dicerl functions as a haploinsufficient tumor suppressor. Genes Dev. 23, 2700-2704 (2009).
39. Lambertz, I. et al. Monoallelic but not biallelic loss of Dicer1 promotes tumorigenesis in vivo. Cell Death Differ. 17, 633-641 (2010).

40. Bahubeshi, A., Tischkowitz, M. \& Foulkes, W. D. miRNA processing and human cancer: DICER1 cuts the mustard. Sci. Transl. Med. 3, 111 ps146 (2011).

41. Hill, D. A. et al. DICER1 mutations in familial pleuropulmonary blastoma. Science 325, 965 (2009).

42. Sabbaghian, N. et al. Germline DICER1 mutation and associated loss of heterozygosity in a pineoblastoma. J. Med. Genet. 49, 417-419 (2012).

43. Francia, S. et al. Site-specific DICER and DROSHA RNA products control the DNA-damage response. Nature 488, 231-235 (2012).

44. Viswanathan, S. R. et al. Lin 28 promotes transformation and is associated with advanced human malignancies. Nat. Genet. 41, 843-848 (2009).

45. West, J. A. et al. A role for Lin28 in primordial germ-cell development and germ-cell malignancy. Nature 460, 909-913 (2009).

46. Molenaar, J. J. et al. LIN28B induces neuroblastoma and enhances MYCN levels via let-7 suppression. Nat. Genet. 44, 1199-1206 (2012).

47. Chang, T. C. et al. Lin-28B transactivation is necessary for Myc-mediated let-7 repression and proliferation. Proc. Natl Acad. Sci. USA 106, 3384-3389 (2009).

48. Urbach, A. et al. Lin 28 sustains early renal progenitors and induces Wilms tumor. Genes Dev. 28, 971-982 (2014).

49. Landthaler, M., Yalcin, A. \& Tuschl, T. The human DiGeorge syndrome critical region gene 8 and its $D$. melanogaster homolog are required for miRNA biogenesis. Curr. Biol. 14, 2162-2167 (2004).

50. Lee, Y. et al. The role of PACT in the RNA silencing pathway. EMBO J. 25, 522-532 (2006)

51. Fleige, S. \& Pfaffl, M. W. RNA integrity and the effect on the real-time qRT-PCR performance. Mol. Aspects Med. 27, 126-139 (2006).

52. Sander, J. D. et al. ZiFiT (Zinc Finger Targeter): an updated zinc finger engineering tool. Nucleic Acids Res. 38, W462-W468 (2010).

53. Sander, J. D. et al. Targeted gene disruption in somatic zebrafish cells using engineered TALENs. Nat. Biotechnol. 29, 697-698 (2011).

54. Jackson, E. L. et al. Analysis of lung tumor initiation and progression using conditional expression of oncogenic K-ras. Genes Dev. 15, 3243-3248 (2001).

\section{Acknowledgements}

We acknowledge patients and families who participated in this study. We thank Thomas Tuschl and V. Narry Kim for providing expression constructs, Eric Olson and Stephen Skapek for helpful comments on the manuscript, and Jose Cabrera for assistance with figures. This study was supported by grants from the Children's Medical Center Foundation, Dallas, TX, and a Young Investigator Research Grant from the Society for Pediatric Pathology (to D.R.); from NIH (R01CA120185 and P01CA134292 to J.T.M.; R01CA135731 to J.F.A.); and the Cancer Prevention and Research Institute of Texas (R1008 to J.T.M. and RP110394 to J.F.A.). K.S.C. is a Damon Runyon-Sohn Pediatric Fellow supported by the Damon Runyon Cancer Research Foundation (DRSG-4P-13) and is also supported by the Children's Medical Center Foundation and Physician Scientist Oncology T32 Training Grant 5T32CA136515-03 to the UT Southwestern Harold C. Simmons Comprehensive Cancer Center.

\section{Author contributions}

D.R., K.S.C., Y.L., A.A.S., V.S., T.-C.C. and S.K. performed experimental work. D.R., K.S.C., Y.L., N.J.K., J.S.M., J.E.W., J.T.M. and J.F.A. analysed data. D.R., K.S.C., Y.L., J.T.M. and J.F.A. produced the figures and text. D.R., J.T.M. and J.F.A. provided overall leadership. All authors contributed to the final manuscript.

\section{Additional information}

Accession Codes: Small RNA sequencing data have been deposited in the Gene Expression Omnibus (GEO) Database under the accession code GSE56955. Wholeexome sequence data of tumours for which patients (or their parent/legal guardian) gave consent for the deposit of their information have been deposited in $\mathrm{dbGaP}$ under the accession code phs000780.v1.p1 (http://www.ncbi.nlm.nih.gov/projects/gap/cgi-bin/ study.cgi?study_id=phs000780.v1.p1)

Supplementary Information accompanies this paper at http://www.nature.com/ naturecommunications

Competing financial interests: The authors declare no competing financial interests.

Reprints and permission information is available online at http://npg.nature.com/ reprintsandpermissions/

How to cite this article: Rakheja, D. et al. Somatic mutations in DROSHA and DICER1 impair microRNA biogenesis through distinct mechanisms in Wilms tumours. Nat. Commun. 5:4802 doi: 10.1038/ncomms5802 (2014). 


\section{Publisher Correction: Somatic mutations in DROSHA and DICERT impair microRNA biogenesis through distinct mechanisms in Wilms tumours}

Dinesh Rakheja, Kenneth S. Chen, Yangjian Liu, Abhay A. Shukla, Vanessa Schmid, Tsung-Cheng Chang, Shama Khokhar, Jonathan E. Wickiser, Nitin J. Karandikar, James S. Malter, Joshua T. Mendell \& James F. Amatruda

Nature Communications 5:4802 doi:10.1038/ncomms5802 (2014); Published 5 Sep 2014; Updated 29 Nov 2017

The HTML version of this Article previously published had an incorrect volume number of 2; it should have been 5 . This has now been corrected in the HTML; the PDF version of the paper was correct from the time of publication. 